\title{
Changes in the strength of prepulse inhibition with variation in the startle baseline associated with individual differences and with old age in rats and mice
}

\author{
JAMES R. ISON, G. PETER BOWEN, JAMES PAK, and ELSA GUTIERREZ \\ University of Rochester, Rochester, New York
}

\begin{abstract}
We examined the relationship between variation in the peak level and temporal course of prepulse inhibition (PPI) of the acoustic startle reflex (ASR) and between-subject variation in ASR control baselines. Prepulses were light flashes (for young rats, $N=155$ ), silent gaps in noise (young mice, $N=46$ ), or noise pulses in quiet (young, middle aged, and aged mice, $N=20$ ). Differences in the baseline ASR accounted for up to $99 \%$ of the variance in difference-score measures of PPI (ASRcontrol - ASRprepulse), but not $10 \%$ of the variance in relative measures $[1-(\mathrm{ASRp} / \mathrm{ASR})]$. The temporal course of relative PPI did not differ with baseline ASR, nor did peak values of relative PPI in Experiments 2 and 3, but were greater $(p<.05)$ in low than in high ASRc subgroups in Experiment 1 . These findings (1) indicate that the neural substrates for ASR elicitation and for PPI are largely independent; (2) reveal that, unlike the ASR, PPI does not decline with age; and (3) suggest the outlines of a formal model for interpreting changes in relative versus difference-score measures of PPI when baseline ASR varies.
\end{abstract}

The acoustic startle response (ASR) to an abrupt intense sound burst is inhibited by relatively weak nonreflexogenic stimuli that immediately precede the eliciting stimulus, a phenomenon called prepulse inhibition (PPI: see reviews by Hoffman \& Ison, 1980, and Ison \& Hoffman, 1983). In the present report, we describe how the expression of PPI changes with variation in the strength of the baseline startle reflex, as provided in two experiments by individual differences in the ASR within two large samples of homogeneous subjects, rats in one case and mice in the second, and in a third experiment by comparisons between smaller groups of mice that differed in the amplitude of the ASR because of their differences in age. The rationale for the study was in part methodological and in part conceptual. The importance of PPI is indicated by its appearance in many different species and for a variety of preliminary and startle stimuli, which suggest that it provides some special adaptive benefit-for example, that of improving perceptual or motor performance by preventing the intense startle stimulus from disrupting either the preattentive processing of the initial stimulus (Graham, 1975) or motor activities associated with that stimulus (Ison, Sanes, Foss, \& Pinckney, 1990). Because they affect a fundamental biological process, it is not unreasonable to assert that any variables or manipulations

This research was supported by USPHS Research Grants AG09524 and EY05262, a Center Support Grant to the Center for Visual Science at the University of Rochester, EY01319, and The International Center for Hearing and Speech Research. Correspondence should be addressed to J. R. Ison, Department of Brain and Cognitive Sciences, University of Rochester, Rochester, NY 14627 (e-mail: Ison@bcs.rochester.edu). that affect PPI must have considerable intrinsic interest. (An example of this approach is provided in the development of an animal model using PPI to test certain physiological hypotheses concerning the role of attentional mechanisms in the pathogenesis of human psychosis; see Swerdlow, Braff, Taaid, \& Geyer, 1994, for a recent review of this work.)

Unfortunately, when one of these interesting manipulations happens also to affect the vigor of the baseline startle reflex, the choice of a metric to use for quantifying PPI can be critically important in describing the experimental outcome. Thus, it is known that the ASR is substantially weakened by age (in humans, Ford et al., 1995; in rodents, Krauter, Wallace, \& Campbell, 1981), but the interesting question of whether PPI is similarly affected depends on the choice of the PPI metric; as we will see, one sort of measure indicates that age reduces PPI, while a second indicates that age has no effect on PPI. In addition, the ambiguity that presently surrounds the relationship between reflex strength and PPI may suggest that such questions cannot be usefully investigated even when the several methods for quantifying PPI happen to agree. It could be readily argued, for example, that a consistent age-related difference in PPI results as a secondary consequence of an age-related difference in reflex strength, rather than being a primary effect on the neural mechanisms responsible for inhibition. The present study was intended to clarify these vexing methodological and conceptual problems by describing the relationships between different measures of PPI and the ASR baseline under different conditions and by developing a simple formal model of how the underlying processes responsible for PPI and 
for reflex elicitation might interact in order to generate these empirical relationships.

The typical single experiment on reflex inhibition does not have enough subjects to allow for their division into subgroups of subjects that show extreme differences in their baselines, and the empirical question by itself may not seem to warrant the necessarily large commitment of resources necessary for its answer. Fortunately, on two occasions, we have given standard pretests for PPI to large numbers of subjects prior to their serving in other experiments concerned with sensory function, one set concerned with vision in young rats and the second with audition in young mice. Both experiments examined the temporal course of inhibition with variation in lead time, and allowed us to examine how the expression of PPI and its temporal course might vary with individual differences in the baseline startle reflex. Then, in a third experiment, we examined PPI in groups of mice who differed in their baseline startle responses because of their age.

To anticipate the results of these experiments: Measures of PPI that are based on ratios between control and inhibited conditions are largely, if not entirely, independent of differences in the strength of reflex elicitation, whether these differences arise because of individual variation within a homogeneous group of subjects or because of differences in age. A review of the literature shows that this is a common, but not ubiquitous, outcome, since at least one variable, startle stimulus intensity, does not influence difference-score measures of PPI while having a profound effect on relative score measures. A simple model of the underlying substrates for inhibition and for elicitation is proposed that suggests how variation in one or the other metric with variation in reflex strength can depend on the relative locations within the reflex pathway where these several variables have their effects.

\section{EXPERIMENT 1}

In this experiment, the subjects were young male albino rats of an inbred strain. Though they may be presumed to have a homogeneous genetic constitution, their acoustic startle behaviors were extraordinarily varied, with the means for the subjects at the ends of the distributions being more than an order of magnitude apart. These subjects were later used in a series of experiments on visual deficits in rats (see, e.g., DiLoreto, Ison, Bowen, Cox, \& del Cerro, 1995), and the data reported here were obtained from a common pretest. The prepulse was a brief light flash delivered at various times prior to an intense sound burst.

\footnotetext{
Method

Subjects. The subjects were 155 male Fischer 344 rats, obtained from Harlan Sprague-Dawley, Inc., Altamont, NY, at 6 weeks of age and tested 2-6 weeks later. They were maintained in group cages under conditions of constant temperature and humidity, with a 12:12-h light:dark cycle (lights on at $6 \mathrm{a} . \mathrm{m}$.). Food and water were freely available at all times. Tests were run during the working hours of about 9 a.m. to 5 p.m.
}

Apparatus. The subjects were confined for testing in a tunnelshaped cage constructed of wire mesh $(17 \mathrm{~cm}$ long, $13 \mathrm{~cm}$ wide, and $9 \mathrm{~cm}$ high), which could be mounted on a suspended acrylic platform directly over an attached accelerometer (Model SA-2-300, Statham Laboratories, Beverly Hills, CA). The platform was placed in a sound-attenuating chamber $(35 \times 65 \times 43 \mathrm{~cm})$ inside a singlewalled sound-attenuating room $(1.9 \times 2.8 \times 2.5 \mathrm{~m}$, constructed by the Industrial Acoustics Company, Bronx, NY). The startle stimulus was a $115-\mathrm{dB}$ (SPL) $8-\mathrm{kHz}$ tone, $25 \mathrm{msec}$ in duration, provided by a Krohn-Hite function generator (Model 1000A, manufactured by the Krohn-Hite Corporation, Avon, MA), shaped by an electronic switch to have 5-msec rise and decay times, amplified, then delivered through a high-frequency speaker (Model 075-105C, JBL Sound Inc., Los Angeles). The startle stimulus was measured with a General Radio Company sound-level meter (Model 1561) with an impact noise analyzer (Model 1566-B) and a Type 1560-P7 microphone. The preliminary stimulus was provided by a voltage-regulated fluorescent lamp (Sylvania F4TS-CWX) with near instantaneous onset and offset times; it was housed in a well-insulated box with a double-glazed aperture in order to eliminate the noise made by the bulb at light onset. The light flash was $20 \mathrm{msec}$ in duration and was measured in the cage as $30 \mathrm{fc}$ (about $300 \mathrm{~lm}$ ) with a Spectra Candela X-100 meter (Photo Research Co., Burbank, CA). Stimulus presentation and response recording were under computer control.

Procedure. The rats were first dark-adapted for $1-2 \mathrm{~h}$ before the experimental session, then placed in the test cage in the experimental chamber for $5 \mathrm{~min}$ and given a series of four "warm-up" startle stimuli delivered at 30-sec intervals. Data collection began immediately thereafter. The subjects received trials in which the startle stimulus was delivered alone or was preceded by the 20 -msec light flash at lead times (onset to onset) of 20,40, 70, 110, 160, 220, 320, or $500 \mathrm{msec}$. Each condition was presented in semirandomized order within blocks, so that no condition was repeated until each condition had been presented for an equal number of trials. Ten blocks of trials were given with an average interval between trials of $30 \mathrm{sec}$ (range, 15-45 sec).

Data analysis. Two measures of inhibition were used for statistical analysis. For the first, mean amplitudes in units that were linearly related to volts were calculated within subjects for each stimulus condition, and PPI was quantified by subtracting the means for the various prestimulus conditions from the value of the baseline control ASR (i.e., ASRc - ASRp). The second measure of inhibition used a ratio score to determine the relative response values for each prestimulus condition. Here the mean amplitude within each condition for each animal was expressed as a proportion relative to the mean of that animal's baseline, and the proportion for inhibition subtracted from the proportion obtained in the control condition $[1-($ ASRp/ASRc $)]$. Two types of analysis were run on each of these measures. For the first analysis, the mean amplitude in the baseline control condition was used to separate the animals into subgroups of relatively reactive (the top $20 \%$ on baseline responding) and nonreactive animals (those in the bottom $20 \%$ ). Both difference score and relative response measures were analyzed by mixeddesign analyses of variance (ANOVAs) in which high versus low baseline was the between-subjects variable (group) and prestimulus lead time was the within-subjects variable. The degrees of freedom for the repeated measures were adjusted for nonhomogeneity of between-cell correlations by the Hunyh-Feldt procedure. For the second analysis, the difference scores and the relative scores obtained at the time of peak inhibition were correlated with the baseline control amplitudes across all subjects.

\section{Results and Discussion}

The range of individual differences in baseline startle amplitudes in this large group of inbred rats was considerable. At the extremes, performance varied by a factor 
of about 17 to 1 , with mean amplitudes that ranged from a low of $.48 \mathrm{v}$-units to a high of $8.08 \mathrm{v}$-units. For the least vigorous subject, the startle response was reduced by $0.28 \mathrm{v}$-units at the interval of peak inhibition; for the most vigorous subject, the response was reduced by $5.38 \mathrm{v}$ units, a difference that might suggest that the prepulse was more effective in inhibiting the larger response by a factor of about 19 to 1 . However, when these inhibitory effects were expressed as a proportion of their control values, we note that the largest response was reduced by about $66 \%$, while the smallest response was reduced by about $58 \%$. The similarity of these two proportions suggests that relative inhibition is maintained over extremely large differences in baseline startle strength.

Figure 1 provides the mean response amplitudes across each of the stimulus conditions in the upper graph and the mean relative response values in the lower graph, for the $20 \%$ of the subjects with the most vigorous baseline responses and the $20 \%$ of the subjects with the least vigorous responses. As is typical for these conditions, inhibition increased rapidly beyond the $20-\mathrm{msec}$ lead time to a peak inhibitory value obtained in the group at $70 \mathrm{msec}$, with a gradual recovery in response strength thereafter. The high subgroup had mean baseline amplitudes and mean decrements in response amplitude on inhibited trials that were about six times greater than those obtained in the low subgroup. However, despite their substantial differences both in baseline response strength and in the size
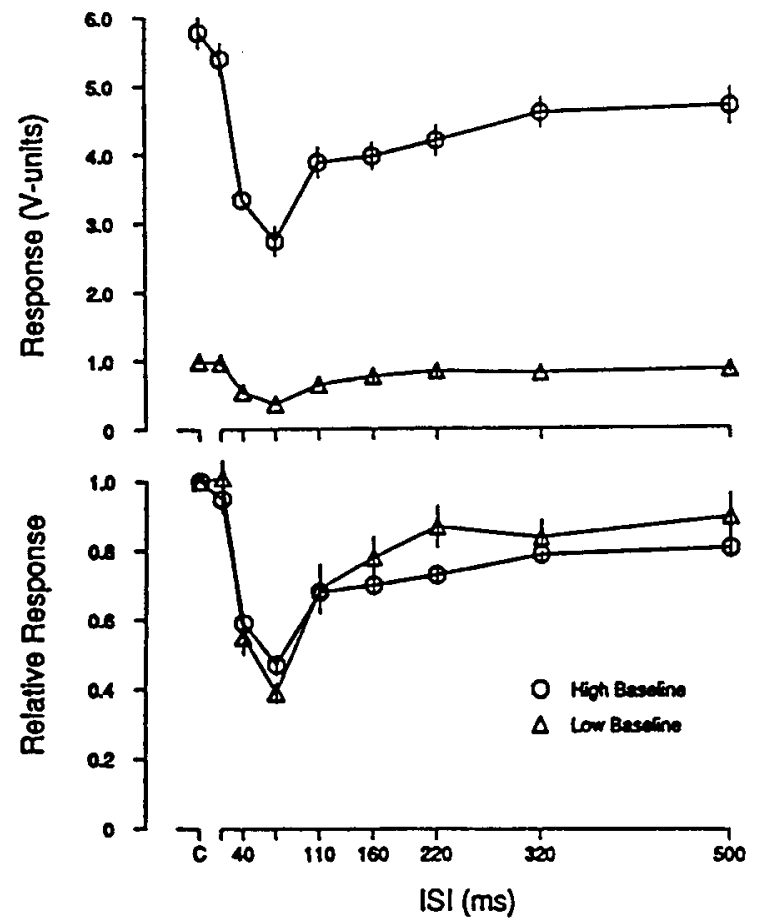

Figure 1. (Above) Mean reflex amplitudes ( $\pm S E M$ ) and (below) mean relative response values $( \pm S E M)$ for baseline startle at $C$ and for the conditions in which a light flash preceded the startle stimulus by the several interstimulus intervals, in the subgroups of rats $(n=31)$ with the highest baseline amplitudes and the lowest baseline amplitudes. of the decrements in response strength produced by the light flash, the peak levels of relative PPI and the temporal functions obtained in the relative measure with changes in prepulse lead time were similar in the two subgroups.

The ANOVA of the difference scores, calculated from the amplitude data presented in Figure 1, resulted in a significant effect for group $[F(1,60)=76.82, p<.0001]$ and in significant effects for both the main effect of lead time $[F(8,480)=59.00, p<.0001]$ and the interaction of group and lead time $[F(1,60)=26.31, p<.0001]$. For the relative measures, the effect of lead time was again significant $[F(7,420)=49.00, p<.0001]$, but in contrast to the effect obtained in the difference-score measures there was no significant effect of group $(F<1)$, indicating that, overall, the two extreme subgroups were not different in this relative measure of PPI. There was, however, a significant interaction between group and lead time $[F(7,420)=2.17, p=.05]$, suggesting the presence of some difference in the details of the temporal course of inhibition between the two groups. Graphically, it appeared that the low-baseline group was more inhibited at the $70-\mathrm{msec}$ peak interval and less inhibited at the longer intervals, those beyond $110 \mathrm{msec}$, though neither of these differences was significant by itself $(p>.05)$.

A correlation of baseline amplitude and peak difference scores obtained between the baseline and the most inhibited response was strongly positive $(r=.88)$, showing, in agreement with the upper portion of Figure 1, that the individual differences in the size of the response decrement produced by a prepulse are determined in large part by the amplitude of the baseline response. The relative measure of inhibition presented a different picture. A scatterplot of baseline amplitude against relative peak inhibition is provided in Figure 2 for all of the subjects in this pretest population. There was a small, but significant, negative correlation between the amplitude of the baseline ASR and peak relative PPI $[r=-.22, t(153)=2.79, p<$ $.01]$. Furthermore, the mean peak of relative PPI in the subgroup with the high ASR values was lower than the peak obtained in the subgroup with low ASR values, at $56 \%$ versus $64 \%[t(60)=2.31, p<.05]$.

We have no certain explanation for these large individual differences in both measures depicted in Figure 2, although it is possible that disparate sensory abilities may account for some small portion of the variance. It is well known that the levels of fluorescent illumination characteristic of well-maintained vivarium quarters produce retinal degeneration in rats (Williams \& Baker, 1980), and light levels within a vivarium must vary from cage to cage, depending on their location. Thus, it is not unreasonable to assume that the subjects in this experiment could vary in their visual sensitivity because of differential exposure to phototoxic levels of light prior to their transfer to our vivarium. The delayed appearance of PPI in rats is a characteristic early sign of retinal degeneration (Ison, Bowen, \& del Cerro, 1992; Wecker \& Ison, 1986), and one small piece of supportive evidence for this hypothesis of individual differences in sensory ability is the observation that about $10 \%$ of the rats showed a delayed 


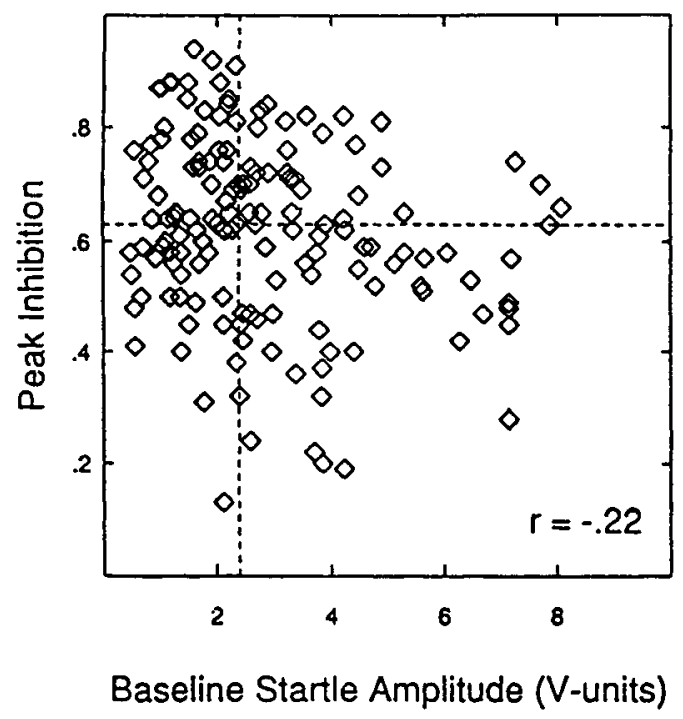

Figure 2. A scattergram of the individual measures of baseline startle amplitude and relative peak PPI produced by the light flash for all subjects $(N=155)$. The vertical line is the median baseline value, the horizontal line is the median value for peak relative PPI.

onset of inhibition. Similarly, some portion of the differences in the baseline ASR may have resulted because of individual differences in auditory sensitivity, perhaps attributable to differential noise exposure during transport or the presence of respiratory infections that invaded the ear. The effects of hearing loss might be expected to be small, because its effects would be most apparent with weak auditory prepulses rather than intense startle stimuli (see Campo et al., 1985). However, to the extent that individual differences in the "subjective intensity" of the startle stimulus had contributed to the differences in the vigor of the startle response, they would also account for the observation that larger responses were associated with somewhat less relative PPI, since this effect is seen when the objective level of the stimulus is varied (see Hoffman \& Ison, 1980). It is possible, also, that differences in the time of day at which the subjects were run could have accounted for some of the variation in ASR amplitude, but this effect would be expected to be small since all were run within the light period (see Davis \& Sollberger, 1971) and variation in time of day would not result in differences in relative PPI (Ison \& Foss, 1997).

\section{EXPERIMENT 2}

Two most interesting outcomes of Experiment 1 are, first, the great extent to which rats that are presumed to have the same genetic heritage differ in their startle baselines and in their level of relative PPI, and, second, the minor effect that even extreme differences in baseline have on relative PPI. The first observation suggests that environmental variables beyond our immediate control, about which we can only speculate, have a large effect on these simple reflex phenomena. The second suggests that two sets of separate and largely independent mechanisms must be responsible for determining the strength of reflex elicitation and reflex inhibition.

In a further analysis of the relationship between individual differences in the strength of PPI and the ASR, we next examined reflex behavior in a group of inbred mice of the CBA/J strain, tested under similar conditions except that the prepulse was a brief silent period in a background noise. These mice, all born and raised within the university vivarium, were used in a series of experiments concerned with the effects of age on temporal acuity (e.g., Walton, Frisina, Ison, \& O'Neill, in press), and the data are taken from a common pretest.

\section{Method}

Subjects. The subjects were 22 male and 24 female CBA/J mice raised in the vivarium at the University of Rochester from a breeding stock originally obtained from the National Institutes of Aging Colony that is maintained by Harlan Sprague-Dawley. They were 2-3 months old at the beginning of testing. All of the mice received evoked potential ABR testing for auditory threshold sensitivity prior to their behavioral tests, and all had normal hearing.

\section{Apparatus and Procedures}

The basic method differed slightly from that described in Experiment 1 . The testing cage was made of acrylic plastic $(7 \mathrm{~cm}$ long, $5 \mathrm{~cm}$ wide, and $4 \mathrm{~cm}$ high) with slotted sides and roof for free sound penetration, and the subjects were run in an anechoic chamber $(84 \times$ $84 \times 84 \mathrm{~cm}$ i.d., manufactured by the Eckel Corp., Cambridge, MA). The startle stimulus was a white-noise burst provided by a wideband noise generator, $115 \mathrm{~dB}$ (SPL), $20 \mathrm{msec}$ in duration, with near instantaneous rise and fall times. The subjects were run in a noise background at $70 \mathrm{~dB}$ (SPL), provided by a second noise generator and delivered through a Panasonic high-frequency leaf tweeter. The spectrum of the startle stimulus varied by no more than $\pm 2 \mathrm{~dB}$ over a range of octave bands centered at $1-32 \mathrm{kHz}$, while the noise background varied by no more than $\pm 6 \mathrm{~dB}$ over a range of $2-100 \mathrm{kHz}$. (These were measured with a $1 / 4$-in. Brüel \& Kjaer microphone, Model 4135, connecting to a measuring amplifier, Brüel \& Kjaer, Model 2610.) The prestimulus was a $10-\mathrm{msec}$ silent period in the gap, presented with near instantaneous fall and rise times, at lead times (onset of the gap to onset of startle) of 10, 15, 20,30, 40,60, 90,110 , and $160 \mathrm{msec}$. Trials were given in 11 blocks, each containing 11 trials, which consisted of 2 baseline control trials and then 1 each of the 9 prestimulus condition trials, at intertrial intervals that averaged $20 \mathrm{sec}$. Only the data of the last 10 blocks were analyzed.

\section{Results and Discussion}

The individual baseline ASR scores in this group of mice varied by a ratio of about 6 to 1 , with mean amplitudes that ranged from a low of $1.34 \mathrm{v}$-units to a high of $8.03 \mathrm{v}$-units. The largest response was reduced by $6.67 \mathrm{v}-$ units at the lead time for peak inhibition, while the smallest response was reduced by $1.06 \mathrm{v}$-units, the difference of about 6 to 1 suggesting greater PPI in the high responder. However, for these mice, the peak effect of the inhibitory stimulus was to reduce the size of the baseline response by about $80 \%$. In agreement with the data ob- 
tained in the two extreme rat subjects, the relative effect of the prepulse was the same in these two mice despite large differences in baseline startle.

Following the same procedure as before, two extreme subgroups were selected from the population, one made up of the (approximately) $20 \%$ of the animals with the highest baselines for their sex, the other, the $20 \%$ with the lowest baselines. (The males had a higher mean baseline response than the females, by about $10 \%$. This was not a significant difference, but 5 males and 5 females were placed in each extreme group so as not to confound sex differences with differences in baseline amplitudes in these analyses.) Figure 3 presents the mean response amplitudes in the upper graph and the relative response amplitudes in the lower graph. The biphasic temporal function that describes the inhibitory effect of a gap in white noise on the startle reflex is that typical of rats and mice, and it agrees with other data in suggesting that two distinct inhibitory processes are involved in these experiments (Ison, 1982; Ison, O'Connor, Bowen, \& Bocirnea, 1991). Response amplitudes in the two subgroups varied by a ratio of about 3 to 1 , as did the response decrements produced by the gap in noise, but the two groups overlapped in their relative response measures. The ANOVA of difference scores pro-
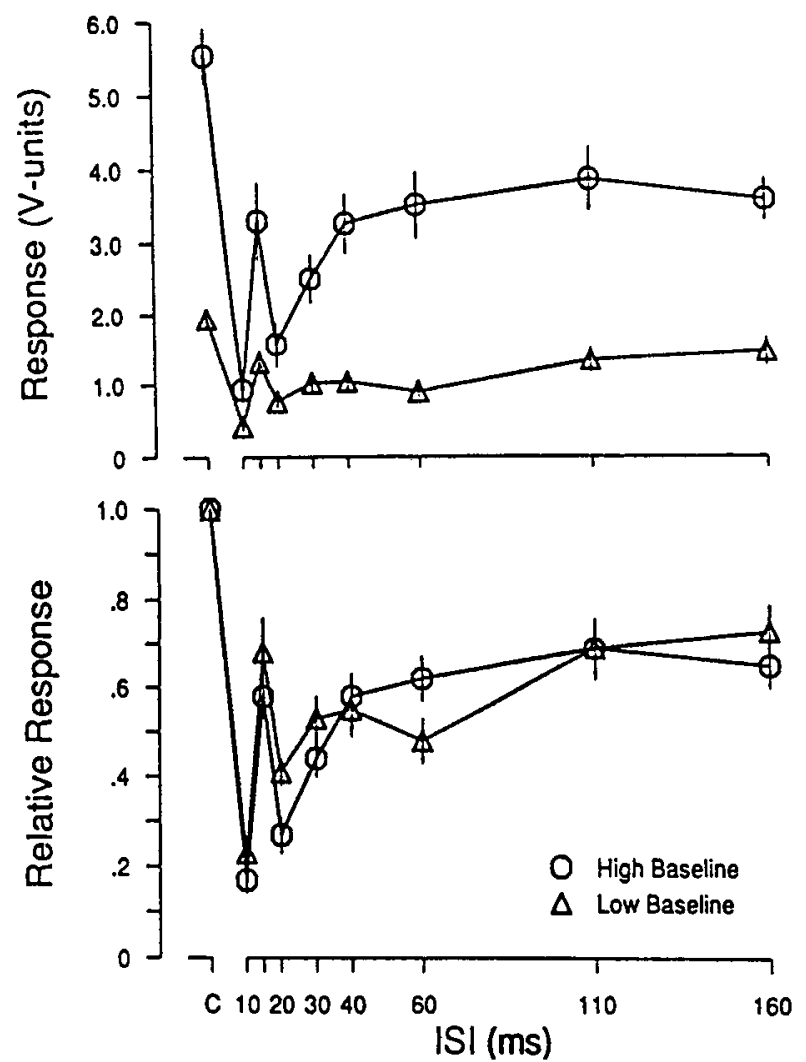

Figure 3. (Above) Mean reflex amplitudes ( $\pm S E M$ ) and (below) mean relative response values $( \pm S E M)$ for baseline startle at $C$ and for the conditions in which a 10-msec gap in noise preceded the startle stimulus by the several interstimulus intervals in the subgroups of mice $(n=10)$ with the highest baseline amplitudes and the lowest baseline amplitudes. vided significant main effects for group $[F(1,18)=66.76$, $p<.0001]$ and lead time $[F(18,144)=49.30, p<.0001]$ and a significant group $\times$ lead time interaction $[F(18,144)$ $=14.43, p<.0001]$. In contrast, the ANOVA of the relative response values found a significant effect of lead time $[F(18,144)=28.22, p<.0001]$ but no main effect for group $(F<1)$. The interaction of lead time and group was not significant $[F(18,144)=1.97, p>.05]$. Across the entire population of mice, the correlation between the baseline response and the difference score for the most inhibited stimulus condition was very strong and positive $(r=.96)$. The relationship between individual differences in peak relative inhibition and in the amplitude of the baseline startle response is depicted in the scattergram of Figure 4. In contrast to the data of the first experiment, here the (nonsignificant) correlation was positive rather than negative, but again the proportion of shared variance in peak inhibition and ASR amplitudes was less than $10 \%$.

The variance in both the ASR baselines and in PPI was reduced in these data as compared with those of Experiment $1[F(155,45)=2.68$ and 7.99 , respectively, $p$ s $<$ $.0001]$. There was still considerable variation in the size of the ASR (with its range of about 6 to 1, compared with about 20 to 1 in Experiment 1), but much less variation for relative PPI (the range was 1.5 to 1 rather than 10 to 1 ). The results of the two experiments were the same, however, in their showing that individual differences in the amplitude of the startle reflex have little consequence for the relative inhibition of the reflex by a preceding stimulus, although they have the major determining effect on difference-score measures of PPI.

\section{EXPERIMENT 3}

To continue the study of the relationship between the strength of PPI and differences in the baseline amplitude of the startle reflex, in this experiment we examined these same variables in groups of inbred mice varying in age from 3 months to about 2 years. Krauter et al. (1981) had previously shown that weak preliminary stimuli do inhibit the startle reflex in old rats of approximately the same age as the mice used in the present experiment, but to a lesser extent than was found in younger rats. They reasonably attributed the age difference in (relative) PPI to age-related decrements in sensory ability, but an additional direct effect of age on PPI could contribute to this effect. Then, further, if the expression of PPI depends in part on the amplitude of the baseline startle reflex, the age effect noted by Krauter et al. could result indirectly because of the smaller startle reactions observed in the older rats. The findings of our first two experiments suggested that the latter possibility was unlikely, but further study of the relationship between PPI and the strength of reflex elicitation in aged subjects was needed.

\section{Method}

Subjects. The subjects were male CBA/J mice obtained from Harlan Sprague-Dawley Inc. at 2 months $(n=5), 7$ months $(n=7)$, and 22-27 months $(n=8)$ of age. 


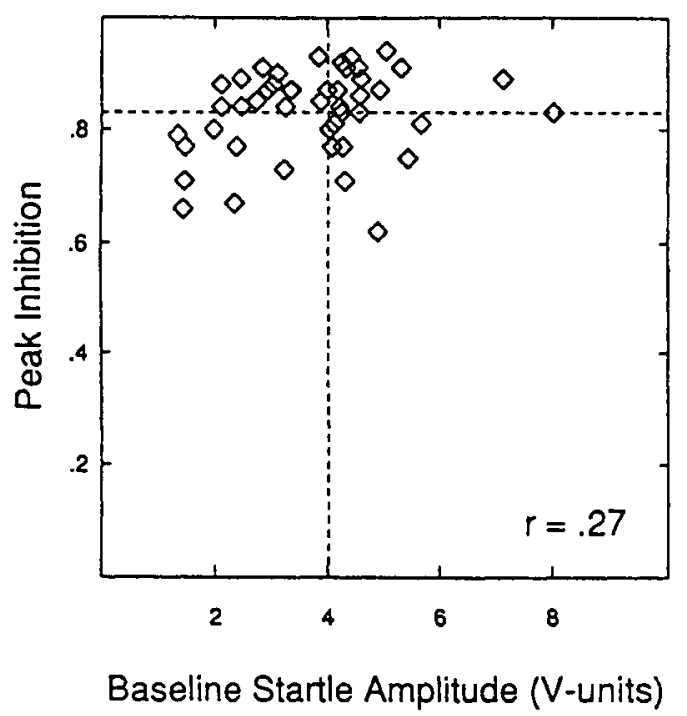

Figure 4. A scattergram of the individual measures of baseline startle amplitude and relative peak PPI produced by the gap in noise for all subjects $(N=46)$. The vertical line is the average baseline value; the horizontal line is the average value for peak relative PPI.

Apparatus and Procedures. The apparatus and the general procedures were the same as those used in Experiment 2 except that the prepulse was a $20-\mathrm{msec}$ burst of white noise with $5-\mathrm{msec}$ rise and decay times set at $70 \mathrm{~dB}$ (SPL) presented against a background of silence, and the startle stimulus also had 5-msec rise and decay times. The interstimulus intervals between the prepulse and the startle pulse were $10,15,20,30,40,60,90,130$, and $190 \mathrm{msec}$, and a block of trials consisted of one each of these conditions plus one startle-alone control trial. Ten blocks of trials were given, preceded by 4 startle-alone trials.

\section{Results and Discussion}

The range of startle baselines was very large when considered across age groups-by a factor of near 20 to $l$ at the extreme. The most vigorous young subject at $6.08 \mathrm{v}-$ units responded with 18 times more force than the least vigorous old subject at $0.33 \mathrm{v}$-units. Nonetheless, this old subject had a peak of relative PPI of $73 \%$, while the peak for the young animal was $70 \%$, strongly suggesting that neither the size of the startle reflex nor the age of the animal has much effect on the relative amount by which the baseline response can be inhibited.

Figure 5 presents the mean response amplitudes for each age group at each condition in the upper portion and the associated mean relative values in the lower portion. The ANOVA of the differences in response amplitude showed a significant effect for age $[F(2,17)=15.80$, $p<.0001]$, for lead time $[F(9,153)=39.20, p<.0001]$, and for their interaction $[F(18,153)=5.13, p<.001]$. For the relative values in the lower figure, the lead time effect was significant $[F(9,153)=57.37, p<.0001]$, but there were no age effects $(F<1)$. Difference score measures of peak PPI were determined in large part by the size of the baseline startle response $(r=.99)$. Figure 6 shows a scatterplot of response amplitude against peak relative
PPI. In contrast to the difference score measure, here PPI was independent of the ASR baseline and the correlation coefficient was zero.

These subjects did not receive threshold tests prior to this experiment, but the oldest group was later found to have an approximately $30-\mathrm{dB}$ upward shift in the threshold for reflex inhibition as compared with the younger groups, in an experiment in which the prepulse varied in level from 20 to $70 \mathrm{~dB}$. This agrees with the results of evoked response ABR thresholds in other CBA mice of the same age, which show a loss of threshold sensitivity across all frequencies of about 20-30 dB. As has been shown previously in human subjects (Reiter \& Ison, 1979), the relationship between PPI and prepulse intensity in mice with a modest sensory loss is flat at low stimulus values and then shows a rapid increase in its strength that looks very much like the classic phenomenon of "loudness recruitment" in hearing-impaired humans (Steinberg \& Gardner, 1937). Reflex elicitation shows the same recruitment effect in hearing-impaired rats (Campo et al. 1985), which makes it unlikely that the age-related differences in the startle baseline seen in the present experiment could be attributed to a mere 20 - to 30 -dB shift in the auditory detection threshold. Ford et al. (1995) came to the same conclusion from their study of moderately hearingimpaired elderly human subjects, having found age-related differences in the ASR even for eliciting stimuli presented

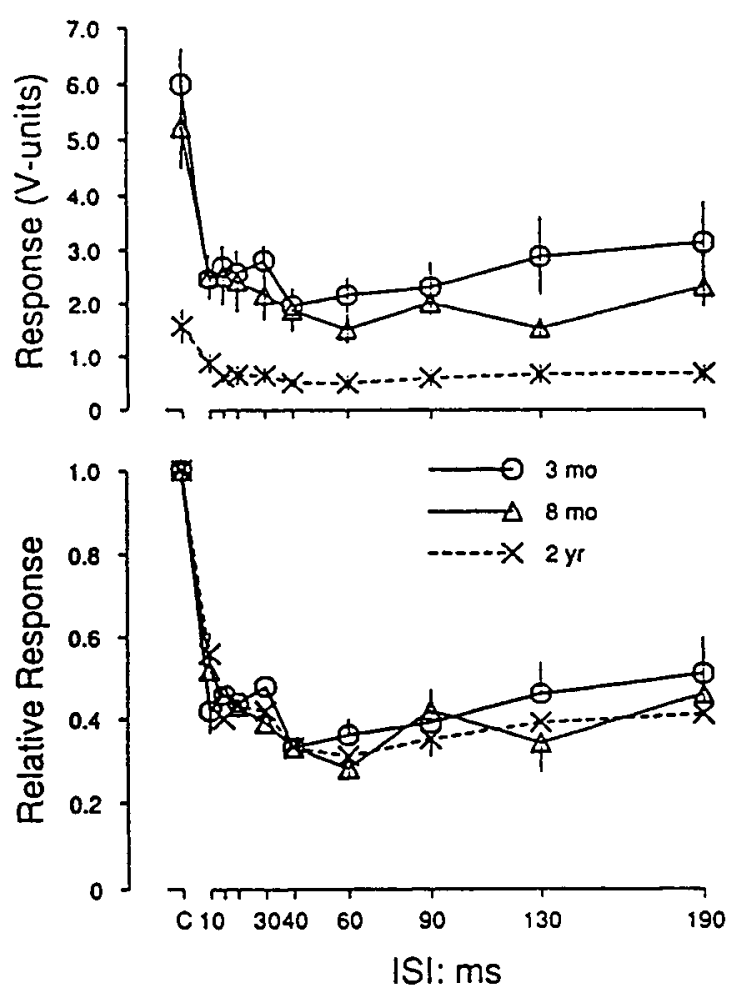

Figure 5. (Above) Mean reflex amplitudes ( $\pm S E M$ ) and (below) mean relative response values $( \pm S E M)$ for baseline startle at $\mathrm{C}$ and for the conditions in which a noise pulse preceded the startle stimulus by the several interstimulus intervals in mice varying in age. 


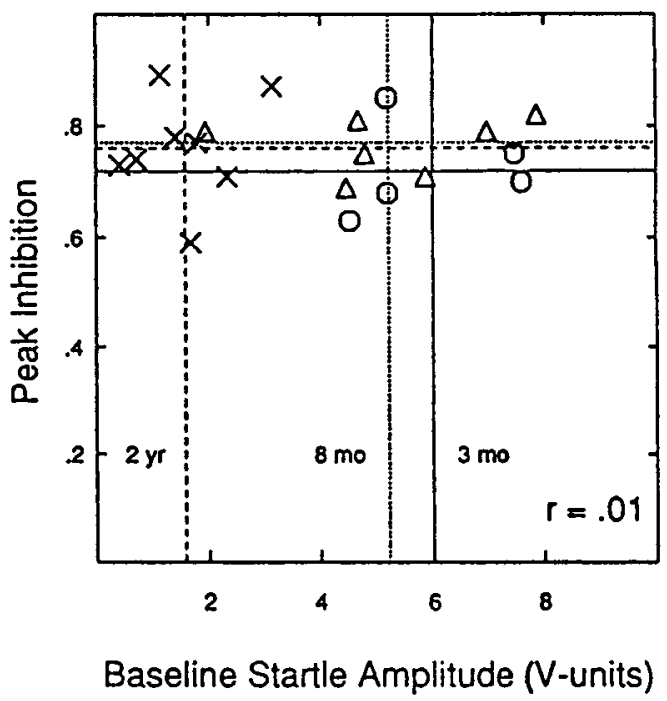

Figure 6. A scattergram of the individual measures of baseline startle amplitude and relative peak PPI produced by the noise pulse for all subjects $(N=20)$. The vertical lines give the average baseline values for each age group; the horizontal lines give the average values for peak relative PPI.

at frequencies for which there was no change in the hearing threshold.

\section{GENERAL DISCUSSION}

The basic finding in these experiments is that relative measures of PPI, quantified by expressing inhibition as a proportional share of the baseline control reflex, did not systematically vary between subjects with high-startle and those with low-startle baselines, whether the differences in the control reflex resulted from uncontrolled individual differences or from differences in the age of the subject. In contrast, individual variation in differencescore measures of PPI were highly correlated with individual differences in the amplitude of the baseline startle reflex, so that the larger the baseline response, the larger the decrement provided by the prepulse. The latter outcome may seem to be a mathematical necessity rather than an empirical finding, simply because it is impossible for the low responder to have the large differences between control and inhibited responses that are present in high responders. To be sure, if the "normative effect" for inhibition were taken as the difference between control and inhibition for the high responder, low responders would necessarily have "below-zero" scores on inhibited trials, which are not physically possible. But, still, if the high value of difference-score inhibition typical of the high-responding subject were to be subtracted from the control value of the low-responding subjects, the temporal function for inhibition in the latter subjects would be expected to be massively distorted. It would be expected that, for these low-responding subjects, relative inhibition would reach $100 \%$ at brief interstimulus lead times and would remain at $100 \%$ until the inhibitory effect obtained in the subjects with the high control responses had waned considerably from its peak. Instead, the time course of relative inhibition was virtually identical in these subgroups of subjects regardless of the size of the control reflex response. These considerations, plus the general observation that peak relative scores are virtually identical across very large differences in the startle baseline, suggest that the fundamental mathematical operation provided by reflex inhibition is that of division rather than subtraction, because it produces a proportional decrement in the amplitude of the reflex regardless of its strength rather than subtracting a constant amount from the control response.

For several other different variables that affect the size of the baseline reflex, including differences in the circadian rhythm (Ison \& Foss, 1997), the presence of other inhibitory stimuli (Ison, Zuckerman, \& Russo, 1975), and muscle strength (Fechter \& Young, 1983), the common effect of a prepulse is also to reduce that response by a constant proportion and to subtract a greater absolute amount from a large as opposed to a small baseline response. The effect of these variables agrees, then, with the effects of individual differences in ASR strength and with the effect of age on ASR strength. But it is critical to note that this otherwise common outcome is never obtained when variation in ASR strength is produced by differences in the intensity of the eliciting stimulus (as reviewed in Hoffman \& Ison, 1980). Instead, in experiments of this type, the effect of the prepulse is to subtract a constant amount from the various responses that vary in size, and, therefore, to provide for larger relative PPI for small than for large baseline responses. (A similar effect on relative PPI was found Experiment 1, it will be recalled. Although the effect was small, and the constant subtractive relationship was not at all apparent in these data, the effect was suggestive that some of the variance in the size of the ASR resulted from individual differences in the subjective intensity of the startle stimulus.)

What could it mean that one variable affects the size of the baseline startle reflex but leaves the relative measure of inhibition intact while a second has an equal effect on the size of the baseline startle reflex but leaves the difference-score measure of inhibition intact? Clearly there must be some important difference in the way that their apparently similar effects on the ASR are achieved, and this difference must also determine the form of the mathematical process by which the excitatory and inhibitory processes that control reflex behavior interact. The initial experiments showing that variation in startle stimulus level did not affect difference-score measures of PPI encouraged the view that the basic mathematical relationship between excitation and inhibition was that of subtraction (e.g., in Hoffman, Marsh, \& Stitt, 1980), and, despite the presence of other variables showing that multiplication by a constant rather than subtraction by a constant was the more frequent effect, this model may be fundamentally correct. But it is now clear that the "sub- 
tractive interaction" of the reflex-eliciting and reflexmodifying pathways proposed in Hoffman and Ison (1980) needs now to incorporate both multiplication and subtraction as permissible mathematical operations, together with assumptions about where these operations interact within the reflex pathways to generate these different patterns of reflex elicitation and reflex inhibition.

In thinking about the outlines of a more complex formal model, it seemed important that startle stimulus intensity, which maintains difference scores but alters relative measures of PPI, must most certainly influence neural activity at the very beginning of the reflex arc, at the level of sensory input, while, in contrast, variation in muscle weakness, which affects difference scores but not relative measures of PPI, must most certainly influence neuromuscular activity at the very end of the reflex arc. As shown in the present work, variation in the age of the subject results in the maintenance of relative measures of PPI, and again the effects of age on the startle reflex seem to be more readily attributed to age-related differences in neuromuscular activity than to sensory activity, given the substantial loss of fast motor units in aging animals (e.g., Pettigrew \& Noble, 1991) as compared with only modest changes in auditory thresholds. The similar circadian effects on reflex elicitation and PPI also result in part at least from changes in activity toward the end of the reflex arc (Frankland \& Ralph, 1995). These observations suggest the reasonable working hypothesis that if a variable affects the reflex-eliciting pathway before it is joined by the inhibitory pathway, the effect of that variable on behavior will be to vary the reflex baseline and maintain difference scores for PPI, whereas, in contrast, if a variable affects the reflex pathway after the subtractive combination of excitation and inhibition, its effect will be to vary the reflex baseline and maintain relative scores for PPI. (Of course, the model necessarily indicates that if a variable affects the inhibitory pathway before it joins the eliciting pathway, the reflex baseline would be maintained but both relative and difference-score measures of PPI would be changed. The obvious variable that has this effect is prepulse intensity.) These considerations agree with the intuition that the neural mechanisms responsible for arithmetic computations could be realized most simply if the site for the subtractive convergence of the neural pathways for reflex elicitation and reflex inhibition came before the site at which neural multiplication was imposed on the now common reflex pathway.

The last point to be made is that the data on which our calculations are based are measures of the force that the subject exerts on the cage in response to the intense sound burst. One should no doubt be surprised that this physical measure, expressed in voltage units, can enter into these precise mathematical relationships of addition and subtraction and multiplication and division with great consistency and accuracy, when the subject is variously exposed to experimental conditions that may include changes in the intensity of the startle stimuli and the prepulse, time of day, and chemicals that induce muscle weakness, or when the subjects vary in age or in the basically unknown conditions that yield individual differences between animals. In other work, we have shown that these voltage measures are proportional to electromyographic recordings of startle-induced neuromuscular activity in the forelimbs of the rat (Hammond, McAdam, $\&$ Ison, 1972). The simplicity of the mathematics of the inhibitory combination of prepulses and startle pulses under various conditions indicates that underlying these electromechanical or electrophysiological measures of reflex elicitation and reflex inhibition are neural mechanisms whose interactive physiological operations follow simple linear rules.

\section{REFERENCES}

CAmpo, A., del Cerro, M., Foss., J. A., Ison, J. R., OrR, J. L., WArren, P. H., \& MONJAN, A. A. (1985). Impairment in auditory function follows perinatal viral infection in the rat. International Journal of Neuroscience, 27, 85-90.

Davis, M., \& Sollberger, A. (1971). Twenty-four hour periodicity of the startle reflex in rats. Psychonomic Science, 25, 37-39.

Diloreto, D., Ison, J. R., Bowen, G. P., Cox, C., \& del Cerro, M. (1995). A functional analysis of the age-related degeneration in the Fischer 344 rat. Current Eye Research, 14, 303-310.

FeChTER, L. D., \& YounG, J. S. (1983). Discrimination of auditory from nonauditory toxicity by reflex modulation audiometry: Effects of triethyl tin. Toxicology \& Applied Pharmacology, 70, 216-227.

Ford, J. M., Roth, W. T., ISAacks, B. G., White, P. M., HoOd, S. H., \& Pfefferbaum, A. (1995). Elderly men and women are less responsive to startling noises: N1, P3 and blink evidence. Biological Psychology, 39, 57-80

Frankland, P. W., \& RalPh, M. R. (1995). Circadian modulation in the rat acoustic startle circuit. Behavioral Neuroscience, 109, 43-48.

Graham, F. K. (1975). The more or less startling effects of weak prestimulation. Psychophysiology, 12, 238-248.

Hammond, G. R., MCAdam, D. W., \& Ison, J. R. (1972). Effects of prestimulation on the electromyographic response associated with the acoustic startle reaction in rats. Physiology \& Behavior, 8, 535-537.

Hoffman, H. S., \& Ison, J. R. (1980). Reflex modification in the domain of startle: I. Some empirical findings and their implications for how the nervous system processes sensory input. Psychological Review, 87, 175-189.

Hoffman, H. S., Marsh, R. R., \& Stitt, C. L. (1980). Tests of a principle of reflex modification: Modification of the human eyeblinkreflex is independent of the intensity of the reflex-eliciting stimulus. Animal Learning \& Behavior, 8, 81-84.

IsoN, J. R. (1982). Temporal acuity in auditory function in the rat: Reflex inhibition by brief gaps in noise. Journal of Comparative \& Physiological Psychology, 96, 945-954.

Ison, J. R., BowEN, G. P., \& DEL CERRO, M. (1992). Impoverished stimulus input does not simulate the slowed visual kinetics of retinal damage. Investigative Ophthalmology \& Visual Science, 33, 104-110.

Ison, J. R., \& Foss, J. A. (1997). Coordinate diurnal variation in the strength of startle elicitation and of startle modification in the rat. Psychobiology, 25, 158-162.

Ison, J. R., \& Hoffman, H. S. (1983). Reflex modification in the domain of startle: II. The anomalous history of a robust and ubiquitous phenomenon. Psychological Bulletin, 94, 3-17.

Ison, J. R., O'Connor, K., Bowen, G. P., \& BocirneA, A. (1991). Temporal resolution of gaps in noise by the rat is lost with functional decortication. Behavioral Neuroscience, 103, 33-40.

Ison, J. R., Sanes, J. R., Foss, J. A., \& Pinckney, L. A. (1990). Facilitation and inhibition of the human startle blink reflex by stimulus anticipation. Behavioral Neuroscience, 104, 418-429. 
Ison, J. R., Zuckerman, M., \& Russo, J. M. (1975). Combination rules for inhibitory stimuli. Journal of Experimental Psychology: Animal Behavior Processes, 1, 318-325.

Krauter, E. E., Wallace, J. E., \& Campbell, B. A. (1981). Sensorymotor function in the aging rat. Behavioral \& Neural Biology, 31, 367-392.

Pettigrew, F. P., \& Noble, E. G. (1991). Shifts in rat plantaris motor unit characteristics with aging and compensatory overload. Journal of Applied Physiology, 71, 2363-2368.

ReITER, L. A., \& Ison, J. R. (1979). Reflex modulation and loudness recruitment. Journal of Auditory Research, 19, 201-207.

Steinberg, J. C., \& Gardner, M. B. (1937). The dependence of hearing impairment on sound intensity. Journal of the Acoustical Society of America, 9, 11-23.

Swerdlow, N. R., Braff, D. L., TaAid, N., \& Geyer, M. A. (1994). As- sessing the validity of an animal model of deficient sensorimotor gating in schizophrenic patients. Archives of General Psychiatry, 51, 139-154.

Walton, J. P., Frisina, R. D., Ison, J. R., \& O'Neill, W. E. (in press). Neural correlates of behavioral gap detection in the inferior colliculus of the young CBA mouse. Journal of Comparative Physiology $B$. WECKER, J. R., \& ISON, J. R. (1986). Visual function measured by reflex modification in rats with inherited retinal dystrophy. Behavioral Neuroscience, 100, 679-684.

WILliams, T. P., \& BAKER, B. N. (1980). The effects of constant light on visual processes. New York: Plenum.

(Manuscript received June 26, 1995; revision accepted for publication March 3, 1997.) 